\title{
Cellular Protection of SNAP-25 against Botulinum Neurotoxin/A: Inhibition of Thioredoxin Reductase through a Suicide Substrate Mechanism
}

\author{
Hajime Seki ${ }^{\dagger}$, Song Xue ${ }^{\dagger}$, Sabine Pellett ${ }^{\S}$, Peter Šilhár $^{\dagger}$, Eric A. Johnson $§$, and Kim D. \\ Janda ${ }^{\dagger, \ddagger}$ \\ tDepartments of Chemistry and Immunology and The Skaggs Institute for Chemical Biology, \\ 10550 North Torrey Pines Road, La Jolla, California 92037, United States \\ ‡Worm Institute for Research and Medicine (WIRM), The Scripps Research Institute, 10550 North \\ Torrey Pines Road, La Jolla, California 92037, United States \\ $\S$ Department of Bacteriology, University of Wisconsin, 1550 Linden Drive, Madison, Wisconsin \\ 53706, United States
}

\begin{abstract}
Botulium neurotoxins (BoNTs) are among the most lethal toxins known to man. They are comprised of seven serotypes with BoNT/A being the most deadly; yet, there is no approved therapeutic for their intoxication or one that has even advanced to clinical trials. Botulinum neurotoxicity is ultimately governed through light chain (LC) protease SNARE protein cleavage leading to a loss of neurotransmitter release. Pharmacological attempts to ablate BoNT/A intoxication have sought to either nullify cellular toxin entry or critical biochemical junctions found within its intricate mechanism of action. In these regards, reports have surfaced of nonpeptidic small molecule inhibitors, but few have demonstrated efficacy in neutralizing cellular toxicity, a key prerequisite before rodent lethality studies can be initiated. On the basis of a lead discovered in our BoNT/A cellular assay campaign, we investigated a family of $N$ hydroxysuccinimide inhibitors grounded upon structure activity relationship (SAR) fundamentals. Molecules stemming from this SAR exercise were theorized to be protease inhibitors. However, this proposition was overturned on the basis of extensive kinetic analysis. Unexpectedly, inhibitor data pointed to thioredoxin reductase (TrxR), an essential component required for BoNT protease translocation. Also unforeseen was the inhibitors' mechanism of action against TrxR, which was found to be brokered through a suicide-mechanism utilizing quinone methide as the inactivating
\end{abstract}

\footnotetext{
H.S. and S.X. contributed equally to this work.

Author Contributions

The authors declare no competing financial interest.

Supporting Information

The Supporting Information is available free of charge on the ACS Publications website at DOI:10.1021/jacs.5b12929.

- Synthesis of the compounds, cellular and biochemical assay protocols, results, and data analysis (PDF)

- Mascot search results for protein identification with probe 31 (PDF)

- Mascot search results for protein identification with control probe 32 (PDF)
} 
element. This new series of TrxR inhibitors provides an alternative means to negate the etiological agent responsible for BoNT intoxication, the $\mathrm{LC}$ protease.

\section{Introduction}

Botulinum neurotoxins are a family of neurotoxins produced by $C$. botulinum, which upon intoxication of neurons may result in a potentially fatal disease called botulism. The neurotoxin is comprised of a $150 \mathrm{kDa}$ single chain peptide composed of $100 \mathrm{kDa}$ heavy chain (HC) and $50 \mathrm{kDa}$ light chain (LC).(1) The HC and LC are linked by a disulfide bond (S-S) and are essential components for BoNT's mechanism of action.(2) On the whole, BoNT's toxicity has been evolutionarily honed exploiting a series of unique steps initiated through neuronal cellular entry. Thus, toxin's selective entrance occurs at the peripheral neuromuscular junction via $\mathrm{HC}$ recognition of the ganglioside and synaptic vesicle glycoproteins, (3) leading to internalization of the toxin via receptor-mediated endocytosis. The $\mathrm{HC}$ within the endosome, upon acidification of the lumen, facilitates translocation of the LC into the neuronal cytosol by generating a channel inside the endosomal membrane.(4) Once released, the LC is now armed for SNARE protein cleavage, thereby blocking synaptic vesicle exocytosis and ultimately release of acetylcholine.(4)As a result, neurotransmissions are ceased causing a neuromuscular blocking effect, which depending on the severity can be fatal. There are seven BoNT serotypes (A-G), wherein A, B, and E are toxic to humans.(1) Notably, SNARE protein-toxin targeting differs between serotypes; thus, BoNT/A and E cleave synaptosome-associated protein 25 (SNAP-25), whereas BoNT/B utilizes synaptobrevin as its substrate of choice.(1)

As stated, vide supra, botulinum neurotoxin acts by halting the release of acetylcholine, which ultimately causes neuromuscular blockade.(5) At the clinical level, this mechanism of action has laid the foundation for the development of the toxin as a highly sought after therapeutic for treatment of such maladies as chronic pain, focal dystonias, spasticity, and hyperkinetic facial lines to name a few, (6) and while BoNT is of substantial clinical value it is this very same mechanism of action that has raised concerns of its use as a weapon of mass destruction.(7) In the past two decades, acts of terrorism continue to remind us of the very real and direct impact of this scourge on civil society.(1) Moreover, the Center for Disease Control and Prevention (CDC) considers botulinum neurotoxin as one of six category A agents.(8) Ultimately, to address a BoNT threat, both fundamental as well as therapeutic studies are needed. In terms of the latter, present treatments for BoNT/A intoxication rely on antitoxin administration and vaccination, which can prevent cellular internalization of toxin during its circulation.(9) However, this approach loses traction once the toxin is internalized within a cell. Currently, there are no approved pharmacological antagonists once BoNT/A cellular intoxication takes place.

A growing number of studies have been conducted with the goal of precluding BoNT/A SNAP-25 destruction, and these have been mainly focused upon the inhibition of the LC protease.(10)BoNT/LC is a $50 \mathrm{kDa}$ zinc-dependent metalloprotease whose sole purpose is the cellular crippling of acetylcholine release. Pharmacologically then, it is logical to develop inhibitors against this protease, given that LC is the etiological agent responsible for 
botulism.(11) Over the past decade, our laboratory has devoted tremendous efforts to discover various LC inhibitors as represented by the active-site prototype zinc chelator, 2, 4dichlorocinnamic hydroxamic acid (1), which is competitive with the SNAP-25 substrate (Figure 1).(12) We have also communicated various allosteric modulators, $\alpha / \beta$-exosite inhibitors such as chicoric acid, lomofungin, and dyngo-4a.(13)Dyngo-4a serves both as a dynamin inhibitor, blocking BoNT/A's cellular entry, and as a LC protease inhibitor, demonstrating the first polypharmacological molecule against BoNT/A.(13c)

One of the significant challenges in targeting the BoNT/A LC has been an unanticipated disconnection between the enzyme and cellular assays. Briefly stated, it has been formidable to inhibit the cellular LC even though sufficient inhibitor potency has been confirmed in LC enzyme assays. We believe this to be in part due to the exceptionally large interacting surface area between the protease and its substrate, SNAP-25. Traditionally, modulation of such protein-protein interactions with a small molecule has proven to be no trivial task.(11) Furthermore, it has been suggested that the biologically relevant form of the LC enzyme is distinct from recombinant LCs used in current assays, due to cellular modifications by host proteins.(14)

\section{Results and Discussion}

\section{Cellular Assay Screen for BoNT/A Inhibitors and SAR Investigations}

Despite the undeniable challenges, the enormous value of an effective BoNT/A inhibitor urged us to look for new chemical scaffolding. Although we previously obtained some success using a prodrug approach, leveraged through carbamate-protected hydroxamic acids, to counteract BoNT/A LC in the cell, we also noted that this design strategy still needed improvement.(15) As an alternative means to enhance the potency of LC inhibitors, we turned our attention to Cys165, which is embedded within the active site of the BoNT/LC (highlighted with yellow in the crystal structure, Figure 1).(16) Moreover, Dive and coworkers reported that when this Cys residue was covalently modified, catalysis was stopped and the enzyme was inactivated.(17) This observation prompted us to prepare a series of compounds based upon our inhibitor 1 that could extend into the space that Cys 165 occupies. However, due to the aforementioned enzyme-cellular disconnect, we decided to initiate these efforts at the cell-based level.

Using a cellular screen, we were guided to $\mathbf{2}$, a protected succinimide derivative of $\mathbf{1}$ (Figures 1 and 2; see the Supporting Information for synthesis and characterization). This succinimide demonstrated SNAP-25 protection with an $\mathrm{EC}_{50}$ value of $63 \mu \mathrm{M}$, and while the potency of $\mathbf{2}$ is modest, we considered this $N$-hydroxysuccinimide as a lead to further develop more efficacious inhibitors.

The protecting group embedded within 2 , a 4-acetoxybenzyl, is known to liberate a quinone methide species via enzymatic cleavage of the acetyl group and subsequent 1, 4-elimination (Figure 3). On the basis of this knowledge, we examined 2 in our SNAPtide assay to determine its inhibitory activity. Surprisingly, however, $\mathbf{2}$ was found to be inactive against BoNT/A LC. Although we were initially confident that $\mathbf{2}$ would be an active site inhibitor, we also examined 2 in our LCMS 66-mer assay. In contrast to the SNAPtide assay, this 
assay is also able to delineate exosite binding and thus a potential allosteric inhibition mechanism. Again, $\mathbf{2}$ was found to be inactive. Because $\mathbf{2}$ presented inhibition in our cellular assay, we assumed that the 4-acetoxybenzyl group was necessary for the membrane permeation, and its liberation to 3 (Figure 2) was occurring via simple acid/base or enzymatic hydrolysis. Either event would produce 3, which we assumed would be responsible for the LC inhibition observed. However, we were surprised to find that $\mathbf{3}$ was inactive in either enzyme assay. In a final attempt to reconcile our observations, we looked to $\mathbf{4}$, the hydrolyzed form of $\mathbf{3}$. This compound also showed poor BoNT/A LC inhibitory activity $\left(\mathrm{IC}_{50}=30 \mu \mathrm{M}\right)$ in the 66 -mer assay. Additionally, the compound posed instability issues in solution, which also made us doubt its veracity as the molecule responsible for cellular BoNT/A inhibition.

Although compounds $\mathbf{2}, \mathbf{3}$, and $\mathbf{4}$ were originally expected to target the active site of BoNT/A's LC, such scaffolding possessing two hydrophobic arenes would clearly impact future development from a solubility standpoint. As a part of our SAR studies, succinimides 5 and $\mathbf{6}$ (Table 1), 3 devoid of two aryl appendages, were synthesized and tested in the cellular assay. Unexpectedly, both molecules showed low micromolar potency, which is virtually a 10 -fold increase in potency from $\mathbf{2}$.

The $N$-hydroxysuccinimide appendage embedded within 6 presented a very simple chemical architecture, yet as shown in Table 1, displayed significant potency, which was clearly unforeseen. As a means to begin to delineate a possible inhibitory mechanism, we first surveyed $N$-hydroxysuccinimide (NHS) and its open-form, 4-(hydroxyamino)-4oxobutanoic acid, in the LC enzyme assay. As anticipated, the two compounds did not demonstrate any inhibition. Again, we decided to reconsider a BoNT/A substrate hypothesis where now $\mathbf{5}$ and $\mathbf{6}$ might be serving as suicide substrates for the BoNT/A protease. Thus, $\mathbf{6}$ was tested in the enzyme assay against BoNT/A LC as well as holotoxin (LC+HC). Unfortunately, however, we were unable to detect any activity.

While the mechanism of inhibition by 6 remained shrouded, we continued our SAR studies to define essential functionalities in $\mathbf{6}$, which we hoped might lead to mechanistic underpinnings for the inhibitory activity seen. As a starting point, $O$-benzyl $N$ hydroxysuccinimide 7 was prepared to examine the importance of acetyl group. This compound showed no inhibition in the cellular assay, indicating that the fragmentation of the 4-acetoxybenzyl moiety is crucial. Next, we synthesized a series of compounds where the $\mathrm{N}$ hydroxysuccinimide structure was altered as seen in $\mathbf{8 , 9}$, and10 (Table 1), and evaluated their cellular activity. Succinimide $\mathbf{8}$ showed no activity, suggesting that the oxygen atom at the benzylic position is required. Moreover, 9 now possessing the piperidine-2, 6-dione scaffolding was found to be about 10 -fold less active, while unconstrained 10 ( $N$-acetyl- $N$ (benzyloxy)acetamide) was also inactive. Last, we examined the importance of the substitution pattern on the aryl ring through the preparation of ortho-quinone methide precursor 11 (Table 1). Again, this compound showed no activity, indicating that para substitution and the resulting para-quinone methide are vital for inhibition.

On the whole, seeing an order of magnitude difference in potency between $\mathbf{6}$ and $\mathbf{9}$, and considering the structures of inactive compounds $\mathbf{7}$ and $\mathbf{8}$, we hypothesized that potency 
differences might reside in the leaving group. To probe this proposition, we looked for alternative leaving groups to the NHS moiety (Table 2). In search of replacements, we recognized that the NHS appendage has been widely employed in amide ligation reactions as it is an excellent activating group of carboxyl functionalities. On the basis of this, additional activating groups engaged in amide couplings were installed. To our delight, structures having strained succinimides (12 and 13), 1-hydroxylbenzotriazole (HOBt, 14), and 1-hydroxy-7-azabenzotriazole (HOAt, 15) all demonstrated virtually equal potency in the cellular assay (Table 2). In an effort to look at a more biologically relevant leaving group, diethyl phosphate $\mathbf{1 6}$ was examined. Gratifyingly, the compound was active with an $\mathrm{EC}_{50}$ value of $12 \mu \mathrm{M}$. We note that $\mathbf{1 6}$ has also been reported as an anticancer agent against several human cancer cell lines.(18) Interestingly, we did not observe any significant toxicity with this compound in hiPSC-derived neurons.

In light of the NHS-congener findings, vide supra, we next turned our focus to the ester functionality found within $\mathbf{6}$. This particular phenolic ester not only presents a hydrolytic liability issue, but it is also nondescript in structure, and therefore a considerable number of esterases could readily hydrolyze this functionality. We envisioned that finding a more durable ester replacement might not only boost the inhibitor potency but also provide additional selectivity toward the target protein(s). The importance here is that if animal studies were to be broached, metabolic stability would be essential as well.

First, ester congeners cyclohexyl ester (17) and $i$-Pr ester (18) were synthesized (Chart 1). These were tested and discovered to possess an inhibitory profile similar to that of $\mathbf{6}$, indicating that ester steric bulk can be tolerated. Next, the importance of the ester itself was evaluated; here we took recourse to carbamate (19), thiocarbamate (20), carbonate (21), and amide (22). Unfortunately, while these structures provided a more stable linkage, they did not protect SNAP-25 from BoNT/A protease cleavage.

As our last endeavor to identify alternative functionalities to the ester, we investigated replacing the ester carbonyl with sulfur and phosphorus moieties. As shown in Chart 1, a total of eight carbonyl replacements (23-30) were prepared. From this series of compounds, only the $t$-butyl sulfinic ester $\mathbf{2 3}$ displayed inhibitory activity with an $\mathrm{EC}_{50}$ value of $21 \mu \mathrm{M}$. Looking to the literature, this result was not all that surprising as sulfinic esters are known to be highly selective toward nucleophilic substitution by thiols.(19) Although this lends little information on specific protein target(s), we still viewed this as valuable knowledge where a thiol/cysteine could be involved in the ester cleavage event, assuming that $\mathbf{6}$ and $\mathbf{2 3}$ share a common protein target. Placing these findings in context, we note quinone methide precursor mastery seen in the seminal publications by Danzin, Widlanski, and others.(20) In an example of phosphatase inhibition, Widlanski and co-workers demonstrated that the rate of quinone methide formation significantly depends on the nature of leaving group, that is, fluoride, chloride, bromide, and acetate.(20c)Benzylic fluoride was found to be an excellent leaving group, whereas benzylic acetate turned out to be a poor leaving group resulting in slow inactivation of the enzyme. These observations are consistent with our hypotheses put forth and assay results found in Tables 1 and 2. 


\section{Target Identification of the Cellular Active Inhibitors}

The challenges of finding an inhibitor's cellular target(s) are undeniably forbidding. Yet, we were buoyed by strong SAR data, which we hypothesized would assist and greatly enhance this overall process. As a starting point, we carefully re-examined our cellular assay. In our assay protocol, neuronal cells are initially incubated with BoNT/A for several minutes, followed by a change of culture medium to remove extracellular toxin, culminating with the addition of the inhibitor to be tested.(15a) Because of this protocol and the known fast toxinuptake by hiPSC-derived neurons, (21) it was an unlikely scenario that our inhibitors were blocking endocytosis of BoNT/A. Furthermore, because our enzyme assay revealed that 6 was inactive against BoNT/A LC, we surmise that our inhibitors are likely to be perturbing the modification of LC by host cytosolic enzymes or the translocation of LC from endosome.

It has been disclosed that BoNT/A is a substrate for Src kinase, which upon phosphorylation drastically augments LC's catalytic activity and thermal stability.(14) To probe whether or not 6prevents BoNT/A's phosphorylation, we screened the inhibitor against a panel of 50 kinases including Src at $20 \mu \mathrm{M}$ (see the Supporting Information). Unfortunately, 6 presented no activity against all kinases examined. Although some unbeknownst cytosolic modification of the BoNT's protease in the cellular milieu could still be evoked, we began to consider that inhibition was linked to the translocation step in BoNT/A's intoxication process.

Upon cellular binding and subsequent endocytosis, BoNT/A enters the neuron as a cargo of the endosome.(22) The endosome within a nerve terminal contains an ATP-dependent proton pump that generates an acidic makeup in the lumen.(4) It is this resulting $\mathrm{pH}$ decrease that ultimately triggers a conformational change in BoNT's HC, exposing hydrophobic domains to create a channel for LC release.(22) Toosendanin, a triterpenoid from traditional Chinese medicine, has been known to neutralize BoNT intoxication.(23) In 2009, we deciphered toosendanin's mechanism of inhibition for both BoNT/A and/E. These studies demonstrated how toosendanin occludes the translocation step via direct interaction with the membrane-embedded HC channel, which ultimately blocks LC release.(24) Parallel studies have also shown bafilomycin A1 to be a selective inhibitor of endosomal ATPase, as it precludes the acidification of the endosome lumen, thus preventing channel formation by the HC in BoNT's translocation step. Simpson and co-workers demonstrated that the efficacy of bafilomycin A1 diminished with time in a mouse phrenic nerve hemidiaphrgam assay.(25) Specifically, bafilomycin A1 presented activity when added to tissue simultaneously or immediately after the addition of BoNT/A, but it displayed reduced activity when added $0.5 \mathrm{~h}$ after the toxin exposure. Similar results were reported by Bavari and co-workers in chicken ventral spinal cord cells.(26) Importantly, these studies establish toosendanin and bafilomycin A1 to inhibit BoNT/A's translocation step. However, once the LC is released into the cytosol, neither compound is effective because translocation is complete.

On the basis of these findings, we designed a similar experiment with $\mathbf{6}$, toosendanin, and bafilomycin A1 using our hiPSC-derived neuron assay (see the Supporting Information for 
assay details). Thus, after toxin exposure, each inhibitor was added at increasing time points ( $0-8 \mathrm{~h}$ delay), and SNAP-25 cleavage was evaluated. As expected, both toosendanin and bafilomycin A1 were initially effective. However, as inhibitor addition was delayed, more SNAP-25 cleavage was detected. Evaluation of $\mathbf{6}$ under these conditions presented an inhibition profile similar to that of toosendanin and bafilomycin A1. This was the first indication that 6's mode of action could be associated with toxin translocation.

Although these translocation studies are noteworthy, it would be still challenging to identify potential targets simply by examining proteins known to be involved in translocation. Furthermore, while much is understood detailing BoNT's mechanism of action, it is still quite possible that not all proteins involved in the toxins translocation have been elucidated. BoNT's translocation progression has been the least studied as compared to other events leading to nerve intoxication. Hence, we gravitated to a direct target identification approach utilizing MS-based proteomics. On the basis of the requirement that the inhibitor must possess an ester linkage and knowledge that its cleavage would release a reactive-covalent quinone methide "tag", we envisioned installation of a "pull-down handle" would be sufficient for protein enrichment and MS/MS analysis. To this end, probe 31, an analogue of 17, was synthesized along with negative control 32, an analogue of 19(Figure 4). Both compounds were tested in the cellular assay before proteomics was to be enacted, and it was confirmed that $\mathbf{3 1}$ provided complete SNAP-25 protection at $20 \mu \mathrm{M}$, and the control $\mathbf{3 2}$ showed no inhibition at $100 \mu \mathrm{M}$ (Figure S2.3).

The proteomics engaged was based on common literature protocols.(27) In short, the probe $(100 \mu \mathrm{M})$ was incubated with cultured neuronal cells for $1 \mathrm{~h}$. Upon cell lysis, protein mixtures were subjected to "click reaction" conditions for biotin attachment to labeled proteins, which were then enriched on streptavidin-agarose beads. Finally, the enriched proteins were digested, and their peptide fragments were analyzed by LC/MS/MS to obtain protein IDs. For this experiment, the mouse neuroblastoma cell (neuro2a) was selected due to its ease of culturing and scale up. As a result, probe $\mathbf{3 1}$ afforded dozens of proteins, most of which were also present when probe $\mathbf{3 2}$ was used, presumably due side reactions and/or nonspecific binding to agarose beads. However, even with these caveats noted, we were able to detect two proteins that were specific to probe 31: thioredoxin reductase 1 isoform 2 and protein disulfide isomerase ERp61 precursor.

The thioredoxin system can be viewed as a ubiquitous cellular hierarchy capable of reducing protein disulfides from archaea to man, consisting of thioredoxin (Trx), thioredoxin reductase (TrxR), and NADPH.(28) Mechanistically, a complex shuttling of electrons allows NADPH and TrxR to reduce Trx, which is responsible for maintaining proteins in their reduced state. In the context of botulinum neurotoxin research, it has been shown that BoNT hijacks the thioredoxin system to cleave the disulfide bond between $\mathrm{HC}$ and LC during the translocation step.(29)Moreover, Montecucco and co-workers have demonstrated that inhibition of Trx and TrxR can lead to SNAP-25 protection in neurons, which was further evaluated in a mouse lethality model using a well-established thioredoxin reductase inhibitor, ebselen.(30) In contrast, protein disulfide isomerase ERp61 precursor is a member of the protein disulfide isomerase (PDI) family. PDI is an essential chaperon that has been shown to augment the folding process of proteins via oxidation of sulfhydryl groups and 
reduction/isomerization of disulfides.(31)Intriguingly, the sequence of ERp61 has high homology to that of TrxR, comprising a similar active site.(31) Thus, it is understandable that a member of the PDI family was identified in the proteomics experiment. It is of note that only a single report on PDI has been disclosed in the context of interchain disulfide reduction of BoNT/A, and in this study it was shown to be incapable of its reduction.(29b)

With this sequence of protein discoveries, it is tempting to conclude that our inhibitors specifically target TrxR and/or PDI through covalent inactivation as suicide substrates. However, caution must be taken because the proteomics experiment merely represents the most abundant proteins that were labeled by probe

1. It is easily imaginable that there are other proteins labeled, but were not found due to the detection limits of MS/MS. An additional sobering thought is that TrxR and PDI are simply major cellular proteins, which could also be a factor defining our proteomic results. Having exercised such caution, however, it is still logical to conjecture that our inhibitors could target thioredoxin reductase, and its inhibition is, at least in part, likely to preclude BoNT/A $\mathrm{LC}$ release from $\mathrm{HC}$ in the translocation step.

\section{More Evidence Implicating TrxR as the Target of Inhibitors 6 and 23}

As a means to further refute or confirm TrxR or PDI as our inhibitor(s) target(s), we evaluated two representative inhibitors (6 and 23) in TrxR and PDI enzyme assays. Both 6 and $\mathbf{2 3}$ demonstrated potent inhibition against thioredoxin reductase, with $\mathrm{IC}_{50}$ values of $0.71 \pm 0.03$ and $5.39 \pm 0.35 \mu \mathrm{M}$, respectively (Figure $5 \mathrm{~A}$ ). However, only $\mathbf{2 3}$ demonstrated inhibition against PDI with an $\mathrm{IC}_{50}$ value of $41.41 \pm 13.37 \mu \mathrm{M}$ (Figure 5B). These results are consistent with the cellular data and labeling experiments, and firmly suggest that TrxR is the target of our inhibitors.

Mammalian thioredoxin reductase is a selenium-dependent flavoprotein, and its active site consists of the characteristic sequence motif GC"Sec"G (Sec = selenocysteine) at the Cterminus and CVNVGC at the N-terminus.(32) The experiment we decided to pose was that under physiological conditions Sec or Cys could act in a nucleophilic manner within the TrxR fold, which would involve an acyl transfer of the carboxylic/sulfinic ester moiety. To test the validity of such an inhibition pattern, a $\mathrm{pH}$-sensitive affinity reagent, BIAM $(\mathrm{N}$ (biotinoyl)- $N$-(iodoacetyl)ethylenediamine), which is known to undergo specific alkylation with Cys or Sec residues, was explored. Because of the difference of reactivity between Sec $\left(\mathrm{p} K_{\mathrm{a}}=5.2\right)$ and Cys $\left(\mathrm{p} K_{\mathrm{a}}=8.3\right)$, (33) only Sec should be labeled at $\mathrm{pH} 6.5$, where both Sec and Cys would be labeled at $\mathrm{pH} 8.5$. TrxR was incubated with $\mathbf{6}$ or 23 at pH 7.2 at $37{ }^{\circ} \mathrm{C}$ for $2 \mathrm{~h}$, and then labeled with BIAM at pH 6.5 or 8.5.(33) The BIAM labeling event was viewed by Western blot with avidin-HRP (Figure 6). Under these conditions at pH 6.5, BIAM labeling intensity was diminished as the concentration of inhibitor increased. At $\mathrm{pH} 8.5$, the overall labeling intensity remained relatively constant as inhibitor concentration increased, although it should be noted that $\mathbf{2 3}$ presented an overall clearer inhibition pattern than $\mathbf{6}$. These findings suggest that both inhibitors have reactivity and fidelity profiles toward selenocysteine and $\mathbf{6}$ has enhanced selectivity to Sec than Cys because additional nonspecific cysteines were modified by 
3. Additional logical steps for inhibitor-protein reactivity profiling led us to look to mass spectrometry for 6-TrxR labeling. However, because of the various mammalian isoforms associated with TrxR (55 to $67 \mathrm{kDa})(34)$ and the low inherent molecular weight of our inhibitors, we were unable to obtain definitive data from either digested fragments or whole protein. This sequence of events led us to synthesize TrxR's active site sequence GCSecG and examine its reactivity toward $\mathbf{6}$. Thus, $\mathbf{6}$ was incubated with the peptide, and the reaction was analyzed by LC/MS. Gratifyingly, new m/zpeaks corresponding to "GCSecG + Ac" and "GCSecG + Ac + quinone methide" adduct were detected (Figure 7; see the Supporting Information for assay details).

Having established TrxR as the protein of relevance for our most potent inhibitors, we next sought to ascertain kinetic parameters of $\mathbf{6}$ and 23 through Ellman's reagent, DTNB (5, 5'dithio-bis-[2-nitrobenzoic acid]) assay. Here, a series of concentrations were tested for each of the two inhibitors when TrxR and DTNB were added to the reaction milieu, while the whole process was monitored at $412 \mathrm{~nm}$. Initial velocity analysis suggested the inhibitors underwent a two-step mechanism, a noncovalent, reversible binding step that was then followed by covalent killing of the enzyme's activity (see the Supporting Information). Further kinetic analysis was conducted by DynaFit4 using a two-step model, (35) granting a $K_{\mathrm{i}}$ of $4.2 \pm 0.3$ and $51 \pm 8 \mu \mathrm{M}$, and a $k_{\text {inact }}$ of $(0.78 \pm 0.09) \times 10^{-3}$ and $(3.2 \pm 0.5) \times 10^{-3} \mathrm{~s}^{-1}$ for $\mathbf{6}$ and $\mathbf{2 3}$, respectively.

From these metrics, what is clear is that 6's acetyl ester has greater affinity to TrxR than $\mathbf{2 3}$ 's $t$-butyl sulfinic ester. However, $\mathbf{2 3}$ displayed a more potent $k_{\text {inact }}$, indicating a greater reactivity of the sulfinic ester, which in turn led to a less selective process between the thioland selenol-residues found within TrxR. On the basis of the summation of all analyses, a credible mechanistic scheme that fits the data acquired is proposed in Figure 8.

Having proposed an acyl transfer mechanistic scheme, we make note that a comparable pathway was reported by Badet and co-workers, where they presented the mechanism-based inactivation of glucosamine 6-phosphate synthase via enzymatic hydrolysis of an amide bond, ultimately liberating thioquinone.(20g) Here, inhibition relied on the enzyme's innate function to hydrolyze glutamine to glutamate. This report led us to query the specificity of our inhibitor, and as a stern test, $\mathbf{6}$ was examined with glutathione peroxidase (GPx), which possesses a selenocysteine motif within its active site, akin to TrxR. As anticipated, $\mathbf{6}$ did not show inhibition against GPx at any of the concentrations examined (Figure S8.1), emphasizing the point that $\mathbf{6}$ does not engage all Sec-containing enzymes.

Although 6 did not engage GPx, promiscuous inhibitor profiling between TrxR and GPx has been previously disclosed, (20f, 33) and has been attributed to active site accessibility between these two enzymes. Thus, it has been established that the selenocysteine motif leveraged within TrxR is C-terminal bound(32) and highly flexible, which provides a rationale for TrxR's broad substrate scope, whereas Sec of GPx is sequestered inside of the enzyme's active site.(36) Considering the small architecture of $\mathbf{6}$, however, it is also conceivable that the initial interaction/binding of $\mathbf{6}$ to the protein plays a critical role, as shown in our two-step model based on the kinetics observed. 
Finally, in light of the thiol-reactive nature of $\mathbf{2 3}$, vide supra, the stability of these inhibitors in a thiol-rich concentric cellular environment needed to be addressed. The sensitivity of the sulfinic ester moiety toward glutathione (GSH), a universal cellular component, has been reported.(19)Hence, we conducted MS-based stability studies on $\mathbf{6}$ and $\mathbf{2 3}$ in various medium using deuterium-labeled compounds as standards. While both $\mathbf{6}$ and $\mathbf{2 3}$ displayed similar stability in PBS and culture medium (see the Supporting Information), a significant difference was observed in $5 \mathrm{mM}$ GSH solution. Inhibitor 6 exhibited approximately an $11 \mathrm{~h}$ half-life; in contrast, $\mathbf{2 3}$ was completely depleted within $0.5 \mathrm{~h}$. These results are readily interpreted as neurons are known to possess an abundance of GSH inside the cell, and $\mathbf{2 3}$ 's sensitivity to GSH would justify the decreased potency seen with $\mathbf{2 3}$.

\section{Conclusion}

In summary, we have disclosed a series of potent inhibitors that protect SNAP-25 from cleavage by BoNT/A, using a cell-based assay screen. Instead of using conventional enzymatic screening, we opted for a direct cellular phenotypic screen, based upon the wisdom that more drug-like molecules would emerge from such an endeavor. At the outset, our inhibitor plan was designed to target the LC of BoNT/A, which was based upon our previous hydroxamic acid research initiative. However, SAR studies revealed that this new series of succinimides was not capable of inhibiting the BoNT/A LC either in their prodrug form or in their deprotected state. We were thus led to ask whether in light of the considerations discussed, vide supra, a protein other than the BoNT/A LC was responsible for inhibitory activity seen. We surmised inhibitory activity was due to either modification of LC by the host or prohibiting translocation of the LC from the endosome. To probe the latter hypothesis, a MS-based proteomics approach was employed, and from these studies thioredoxin reductase 1 was discerned as the likely target. Thioredoxin reductase would seem to be an unsuspecting candidate. However, it has been noted that during the translocation step, BoNT requires thioredoxin reductase to liberate the LC from HC, and we posit that this is the most likely scenario of how our inhibitors protect cellular SNAP-25 from BoNT/A. Remarkably, the inhibition seen resides in a suicide substrate mechanism.

A surfeit of studies have come to light in recent years solely devoted to TrxR, especially in the field of oncology where there are a plethora of reports detailing selective and potent inhibitors of TrxR.(37) However, a vast majority of these inhibitors possess either an electrophilic center, for example, a Michael acceptor ( $\alpha, \beta$-unsaturated system), (38) or a metal ion complex, (39) both capable of trapping the catalytic selenocysteine or cysteine residues. Surprisingly, our inhibitor's profile uses an acyl transfer mechanism, and to the best of our knowledge, TrxR possessing a hydrolase-like function has not been previously disclosed. This mechanistic underpinning requires further comment as there are a number of activity-based chemical probes that are homologus to the inhibitors we have disclosed. These inhibitors were utilized for the sole purpose of esterase profiling of the proteome.(40) In light of TrxR's newly-found acyltransfer activity, it is worth mentioning that such probes might have selected TrxR; however, TrxR's selection was dismissed because of its previously unknown hydrolase properties. Regardless of BoNT serotype, all botulinum neurotoxins require translocation of the neurotoxin's protease. We would surmise that, based on our inhibitor's mechanism of action, these molecules will act as pan-inhibitors of all 
BoNT serotypes. Admittedly, translocation inhibitors are not capable of counteracting BoNT once it takes residence inside of the cell, thus limiting their use as a therapeutic. However, based on the elimination half-life of BoNT/A, a 4-10 h window of opportunity would be available, which at least would put these inhibitors on ground similar to that of antibody therapeutics, (41) thus adding a small molecule pharmacological antagonist to the arsenal of emergency treatment options.

\section{Supplementary Material}

Refer to Web version on PubMed Central for supplementary material.

\section{Acknowledgments}

We thank Jing Yu for the preparation of $\mathbf{5}$, and appreciate Linh Hoang and Minerva Tran in the center for metabolomics at TSRI for valuable assistance in the proteomics stage of the research. We also gratefully acknowledge the Skaggs Foundation and the NIAID AI117878 for their generous financial support. This is manuscript 29261 from The Scripps Research Institute.

\section{References}

1. Burnett JC, Henchal EA, Schmaljohn AL, Bavari S. Nat Rev Drug Discovery. 2005; 4:281-297. DOI: 10.1038/nrd1694 [PubMed: 15803193]

2. (a) Montecucco C, Papini E, Schiavo G. FEBS Lett. 1994; 346:92-98. DOI: 10.1016/0014-5793(94)00449-8 [PubMed: 8206166] (b) Simpson L. Toxicon. 2013; 68:40-59. DOI: 10.1016/j.toxicon.2013.02.014 [PubMed: 23518040]

3. Benoit RM, Frey D, Hilbert M, Kevenaar JT, Wieser MM, Stirnimann CU, McMillan D, Ceska T, Lebon F, Jaussi R, Steinmetz MO, Schertler GFX, Hoogenraad CC, Capitani G, Kammerer RA. Nature (London, U K). 2013; 505:108-111. DOI: 10.1038/nature12732 [PubMed: 24240280]

4. Koriazova LK, Montal M. Nat Struct Biol. 2003; 10:13-8. DOI: 10.1038/nsb879 [PubMed: 12459720]

5. (a) Blasi J, Chapman ER, Link E, Binz T, Yamasaki S, De Camilli P, Sudhof TC, Niemann H, Jahn R. Nature. 1993; 365:160-3. DOI: 10.1038/365160a0 [PubMed: 8103915] (b) Chen F, Kuziemko GM, Stevens RC. Infect Immun. 1998; 66:2420-5. [PubMed: 9596697]

6. Schantz EJ, Johnson EA. Microbiol Rev. 1992; 56:80-99. [PubMed: 1579114]

7. Arnon SS, Schechter R, Inglesby TV, Henderson DA, Bartlett JG, Ascher MS, Eitzen E, Fine AD, Hauer J, Layton M, Lillibridge S, Osterholm MT, O’Toole T, Parker G, Perl TM, Russell PK, Swerdlow DL, Tonat K, Biodefense WGC. Jama-Journal of the American Medical Association. 2001; 285:1059-1070. DOI: 10.1001/jama.285.8.1059

8. Sobel J, Khan AS, Swerdlow DL. Lancet. 2002; 359:874-880. DOI: 10.1016/ S0140-6736(02)07947-3 [PubMed: 11897303]

9. Sobel J. Clin Infect Dis. 2005; 41:1167-73. DOI: 10.1086/444507 [PubMed: 16163636]

10. (a) Kiris E, Burnett JC, Kane CD, Bavari S. Curr Top Med Chem. 2014; 14:2044-2061. DOI: 10.2174/1568026614666141022093350 [PubMed: 25335887] (b) Willis B, Eubanks LM, Dickerson TJ, Janda KD. Angew Chem, Int Ed. 2008; 47:8360-8379. DOI: 10.1002/anie. 200705531

11. Breidenbach MA, Brunger AT. Nature. 2004; 432:925-929. DOI: 10.1038/nature03123 [PubMed: 15592454]

12. (a) Boldt GE, Kennedy JP, Janda KD. Org Lett. 2006; 8:1729-1732. DOI: 10.1021/o10603211 [PubMed: 16597152] (b) Silvaggi NR, Boldt GE, Hixon MS, Kennedy JP, Tzipori S, Janda KD, Allen KN. Chem Biol. 2007; 14:533-542. DOI: 10.1016/j.chembiol.2007.03.014 [PubMed: 17524984] (c) Silhar P, Silvaggi NR, Pellett S, Capkova K, Johnson EA, Allen KN, Janda KD. Bioorg Med Chem. 2013; 21:1344-1348. DOI: 10.1016/j.bmc.2012.12.001 [PubMed: 23340139] 
13. (a) Silhar P, Capkova K, Salzameda NT, Barbieri JT, Hixon MS, Janda KD. J Am Chem Soc. 2010; 132:2868.doi: 10.1021/ja910761y [PubMed: 20158239] (b) Eubanks LM, Silhar P, Salzameda NT, Zakhari JS, Feng XC, Barbieri JT, Shoemaker CB, Hixon MS, Janda KD. ACS Med Chem Lett. 2010; 1:268-272. DOI: 10.1021/ml100074s [PubMed: 20959871] (c) Seki H, Xue S, Hixon MS, Pellett S, Remes Combining Breve M, Johnson EA, Janda KD. Chem Commun (Cambridge, U K). 2015; 51:6226-9. DOI: 10.1039/C5CC00677E

14. (a) FerrerMontiel AV, Canaves JM, DasGupta BR, Wilson MC, Montal M. J Biol Chem. 1996; 271:18322-18325. DOI: 10.1074/jbc.271.31.18322 [PubMed: 8702470] (b) Encinar JA, Fernandez A, Ferragut JA, Gonzalez-Ros JM, DasGupta BR, Montal M, Ferrer-Montiel A. FEBS Lett. 1998; 429:78-82. DOI: 10.1016/S0014-5793(98)00571-7 [PubMed: 9657387] (c) Ibanez C, Blanes-Mira C, Fernandez-Ballester G, Planells-Cases R, Ferrer-Montiel A. FEBS Lett. 2004; 578:121-127. DOI: 10.1016/j.febslet.2004.10.084 [PubMed: 15581628] (d) Toth S, Brueggmann EE, Oyler GA, Smith LA, Hines HB, Ahmed SA. Front Exp Pharmacol Drug Discovery. 2012; 3:102.

15. (a) Silhar P, Eubanks LM, Seki H, Pellet S, Javor S, Tepp WH, Johnson EA, Janda KD. J Med Chem. 2013; 56:7870-7879. DOI: 10.1021/jm400873n [PubMed: 24127873] (b) Seki H, Pellett S, Silhar P, Stowe GN, Blanco B, Lardy MA, Johnson EA, Janda KD. Bioorg Med Chem. 2014; 22:1208-1217. DOI: 10.1016/j.bmc.2013.11.053 [PubMed: 24360826]

16. Bremer PT, Hixon MS, Janda KD. Bioorg Med Chem. 2014; 22:3971-3981. DOI: 10.1016/j.bmc. 2014.06.004 [PubMed: 24984937]

17. Stura EA, Le Roux L, Guitot K, Garcia S, Bregant S, Beau F, Vera L, Collet G, Ptchelkine D, Bakirci H, Dive V. J Biol Chem. 2012; 287:33607-33614. DOI: 10.1074/jbc.M112.396697 [PubMed: 22869371]

18. Kodela R, Chattopadhyay M, Nath N, Cieciura LZ, Pospishill L, Boring D, Crowell JA, Kashfi K. Bioorg Med Chem Lett. 2011; 21:7146-7150. DOI: 10.1016/j.bmcl.2011.09.075 [PubMed: 22001089]

19. Malwal SR, Labade A, Andhalkar AS, Sengupta K, Chakrapani H. Chem Commun. 2014; 50:11533-11535. DOI: 10.1039/C4CC05462H

20. (a) Halazy S, Berges V, Ehrhard A, Danzin C. Bioorg Chem. 1990; 18:330-344. DOI: 10.1016/0045-2068(90)90007-R(b) Myers JK, Cohen JD, Widlanski TS. J Am Chem Soc. 1995; 117:11049-11054. DOI: 10.1021/ja00150a002(c) Myers JK, Widlanski TS. Science. 1993; 262:1451-1453. DOI: 10.1126/science.8248785 [PubMed: 8248785] (d) Stowell JK, Widlanski TS, Kutateladze TG, Raines RT. J Org Chem. 1995; 60:6930-6936. DOI: 10.1021/jo00126a051(e) Bauer H, Fritz-Wolf K, Winzer A, Kuhner S, Little S, Yardley V, Vezin H, Palfey B, Schirmer RH, Davioud-Charvet E. J Am Chem Soc. 2006; 128:10784-10794. DOI: 10.1021/ja061155v [PubMed: 16910673] (f) Citta A, Folda A, Bindoli A, Pigeon P, Top S, Vessieres A, Salmain M, Jaouen G, Rigobello MP. J Med Chem. 2014; 57:8849-8859. DOI: 10.1021/jm5013165 [PubMed: 25313665] (g) Massiere F, BadetDenisot MA, Rene L, Badet B. J Am Chem Soc. 1997; 119:57485749. DOI: $10.1021 / \mathrm{ja} 970254 \mathrm{t}$

21. Whitemarsh RCM, Strathman MJ, Chase LG, Stankewicz C, Tepp WH, Johnson EA, Pellett S. Toxicol Sci. 2012; 126:426-435. DOI: 10.1093/toxsci/kfr354 [PubMed: 22223483]

22. Grumelli C, Verderio C, Pozzi D, Rossetto O, Montecucco C, Matteoli M. NeuroTo xicology. 2005; 26:761-7. DOI: 10.1016/j.neuro.2004.12.012

23. Shi YL, Wang ZF. Acta Pharmacol Sin. 2004; 25:839-48. [PubMed: 15169642]

24. Fischer A, Nakai Y, Eubanks LM, Clancy CM, Tepp WH, Pellett S, Dickerson TJ, Johnson EA, Janda KD, Montal M. Proc Natl Acad Sci U S A. 2009; 106:1330-1335. DOI: 10.1073/pnas. 0812839106 [PubMed: 19164566]

25. Simpson LL, Coffield JA, Bakry N. J Pharmacol Exp Ther. 1994; 269:256-262. [PubMed: 8169833]

26. Ruthel G, Burnett JC, Nuss JE, Wanner LM, Tressler LE, Torres-Melendez E, Sandwick SJ, Retterer CJ, Bavari S. Toxins. 2011; 3:207-217. DOI: 10.3390/toxins3030207 [PubMed: 22069707]

27. Speers AE, Cravatt BF. J Am Chem Soc. 2005; 127:10018-10019. DOI: 10.1021/ja0532842 [PubMed: 16011363]

28. Arner ESJ, Holmgren A. Eur J Biochem. 2000; 267:6102-6109. DOI: 10.1046/j. 1432-1327.2000.01701.x [PubMed: 11012661] 
29. (a) Pirazzini M, Bordin F, Rossetto O, Shone CC, Binz T, Montecucco C. FEBS Lett. 2013; 587:150-155. DOI: 10.1016/j.febslet.2012.11.007 [PubMed: 23178719] (b) Kistner A, Habermann E. Naunyn-Schmiedeberg's Arch Pharmacol. 1992; 345:227-234. DOI: 10.1007/BF00165741 [PubMed: 1570025] (c) Pirazzini M, Zanetti G, Lista F, Binz T, Shone CC, Rossetto O, Montecucco C. Toxicon. 2015; 107:32-6. DOI: 10.1016/j.toxicon.2015.06.019 [PubMed: 26130523]

30. Pirazzini M, Tehran DA, Zanetti G, Megighian A, Scorzeto M, Fillo S, Shone CC, Binz T, Rossetto O, Lista F, Montecucco C. Cell Rep. 2014; 8:1870-1878. DOI: 10.1016/j.celrep.2014.08.017 [PubMed: 25220457]

31. Wilkinson B, Gilbert HF. Biochim Biophys Acta, Proteins Proteomics. 2004; 1699:35-44. DOI: 10.1016/S1570-9639(04)00063-9

32. Gromer S, Johansson L, Bauer H, Arscott LD, Rauch S, Ballou DP, Williams CH Jr, Schirmer RH, Arner ES. Proc Natl Acad Sci U S A. 2003; 100:12618-23. DOI: 10.1073/pnas.2134510100 [PubMed: 14569031]

33. Liu X, Pietsch KE, Sturla SJ. Chem Res Toxicol. 2011; 24:726-36. DOI: 10.1021/tx2000152 [PubMed: 21443269]

34. (a) Holmgren A, Bjornstedt M. Methods Enzymol. 1995; 252:199-208. DOI: 10.1016/0076-6879(95)52023-6 [PubMed: 7476354] (b) Williams CH, Arscott LD, Muller S, Lennon BW, Ludwig ML, Wang PF, Veine DM, Becker K, Schirmer RH. Eur J Biochem. 2000; 267:6110-6117. DOI: 10.1046/j.1432-1327.2000.01702.x [PubMed: 11012662]

35. (a) Kuzmic P. Anal Biochem. 1996; 237:260-73. DOI: 10.1006/abio.1996.0238 [PubMed: 8660575] (b) Schwartz PA, Kuzmic P, Solowiej J, Bergqvist S, Bolanos B, Almaden C, Nagata A, Ryan K, Feng JL, Dalvie D, Kath JC, Xu MR, Wani R, Murray BW. Proc Natl Acad Sci U S A. 2014; 111:173-178. DOI: 10.1073/pnas.1313733111 [PubMed: 24347635]

36. Epp O, Ladenstein R, Wendel A. Eur J Biochem. 1983; 133:51-69. DOI: 10.1111/j. 1432-1033.1983.tb07429.x [PubMed: 6852035]

37. Cai WQ, Zhang LW, Song YL, Wang BL, Zhang BX, Cui XM, Hu GM, Liu YP, Wu JC, Fang JG. Free Radical Biol Med. 2012; 52:257-265. DOI: 10.1016/j.freeradbiomed.2011.10.447 [PubMed: 22064364]

38. Gan FF, Kaminska KK, Yang H, Liew CY, Leow PC, So CL, Tu LN, Roy A, Yap CW, Kang TS, Chui WK, Chew EH. Antioxid Redox Signaling. 2013; 19:1149-65. DOI: 10.1089/ars.2012.4909

39. Gasser G, Ott I, Metzler-Nolte N. J Med Chem. 2011; 54:3-25. DOI: 10.1021/jm100020w [PubMed: 21077686]

40. Hakamata W, Tamura S, Hirano T, Nishio T. ACS Med Chem Lett. 2014; 5:321-325. DOI: 10.1021/ml400398t [PubMed: 24900834]

41. (a) Ravichandran E, Gong Y, Al Saleem FH, Ancharski DM, Joshi SG, Simpson LL. J Pharmacol Exp Ther. 2006; 318:1343-51. DOI: 10.1124/jpet.106.104661 [PubMed: 16782822] (b) Al-Saleem FH, Ancharski DM, Ravichandran E, Joshi SG, Singh AK, Gong Y, Simpson LL. J Pharmacol Exp Ther. 2008; 326:856-63. DOI: 10.1124/jpet.108.136242 [PubMed: 18539649] (c) Cheng LW, Stanker LH, Henderson TD 2nd, Lou J, Marks JD. Infect Immun. 2009; 77:4305-13. DOI: 10.1128/IAI.00405-09 [PubMed: 19651864] (d) Al-Saleem FH, Nasser Z, Olson RM, Cao L, Simpson LL. J Pharmacol Exp Ther. 2011; 338:503-17. DOI: 10.1124/jpet.111.180653 [PubMed: 21586604] 


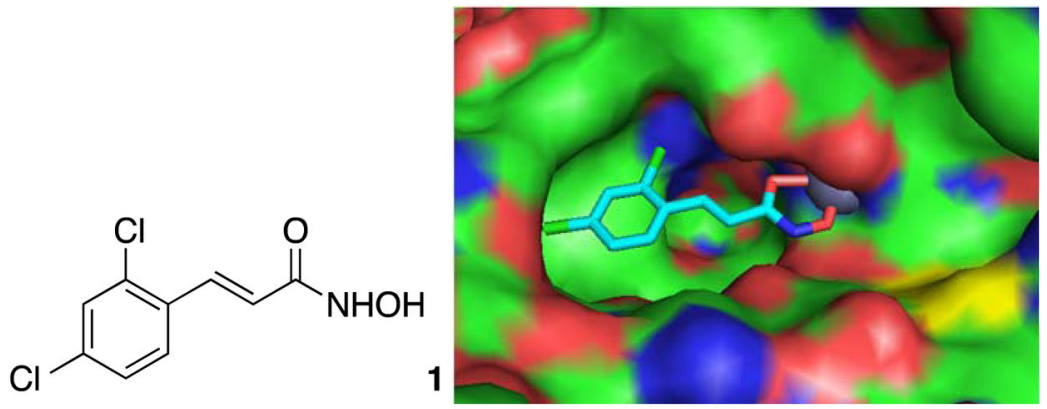

Figure 1.

Structure of inhibitor $\mathbf{1}$ and its crystal structure within BoNT/A's LC active site. 
$\mathrm{Cl}$

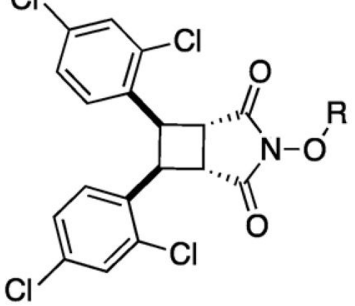

$\mathrm{R}=4$-acetoxybenzyl (2); $\mathrm{EC}_{50}=63 \mu \mathrm{M}$

$=\mathrm{H}(3) ; \mathrm{EC}_{50}>100 \mu \mathrm{M}$

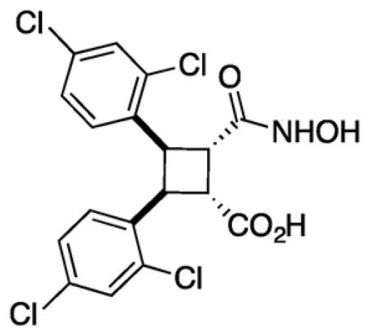

4

Figure 2.

Inhibitor $\mathbf{2}$ selected from cellular screen and analogues ( $\mathbf{3}$ and $\mathbf{4})$ examined in enzymatic and cellular assay. 


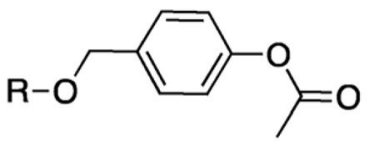

A

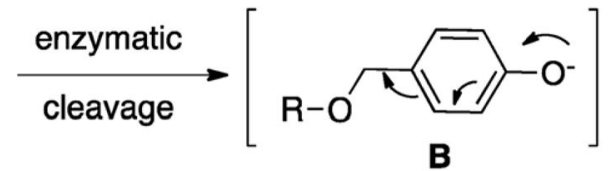

B

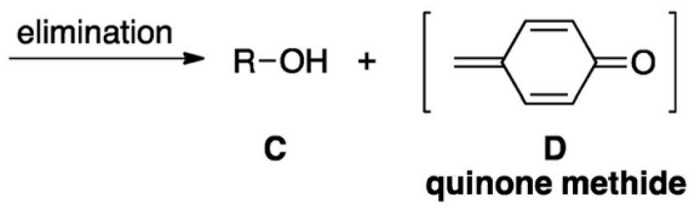

Figure 3.

Mechanism of quinone methide formation. 


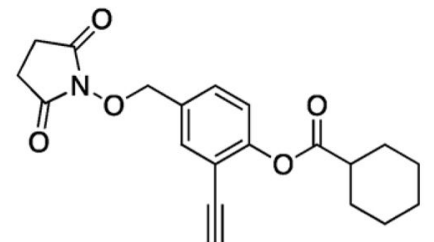

Active probe (31)

Complete SNAP-25 protection at $20 \mu \mathrm{M}$

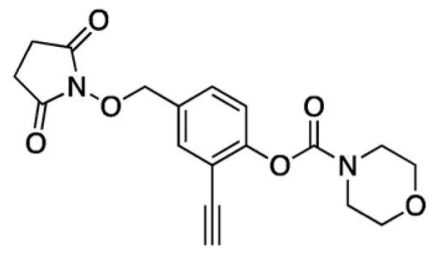

Negative control (32) No SNAP-25 protection at $100 \mu \mathrm{M}$

Figure 4.

Structure of MS proteomic probes $\mathbf{3 1}$ and $\mathbf{3 2}$ (see the Supporting Information for inhibitor profile). 
A

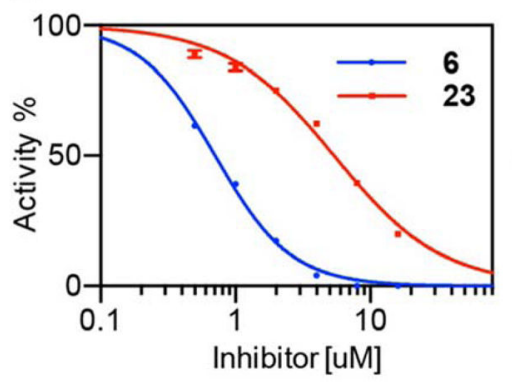

B

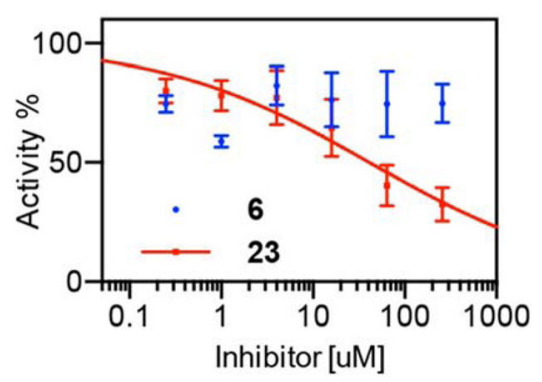

Figure 5.

Inhibition plots $\left(\mathrm{IC}_{50}\right)$ of $\mathbf{6}$ and $\mathbf{2 3}$ with $\operatorname{TrxR}(\mathrm{A})$ or PDI (B). 


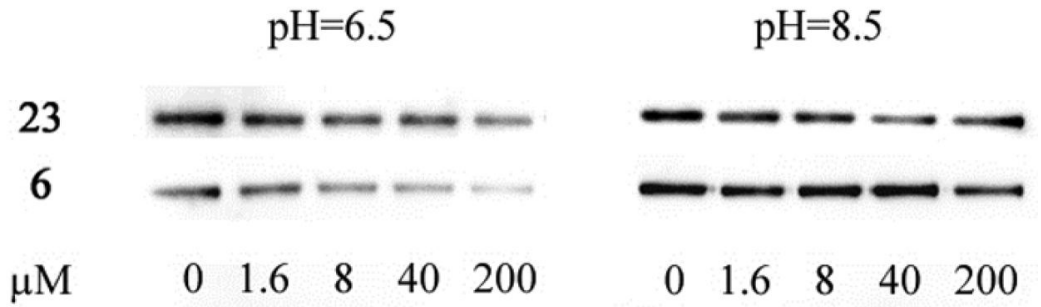

Figure 6.

BIAM labeling of Sec (pH 6.5) and Sec and Cys residues (pH 8.5) (see Figure S5.3 for quantification details). 
A

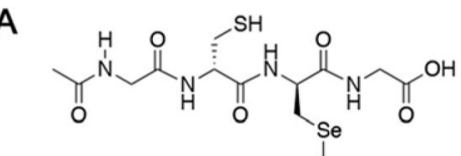

o

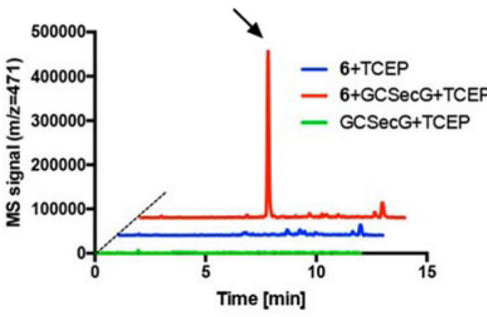

B
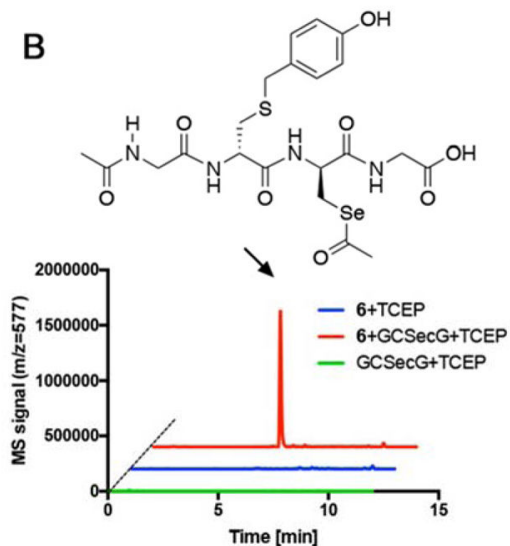

Figure 7.

LC-MS signal of predicted acylpeptide products $(\mathrm{m} / z=471$ (A) or 577 (B)). GCSecG was reduced with TCEP cleaving the Se-S bond and then added to6. After $1 \mathrm{~h}$ of incubation, acylated peptides (A and B) were observed. Samples without $\mathbf{6}$ or peptide were used as controls. 


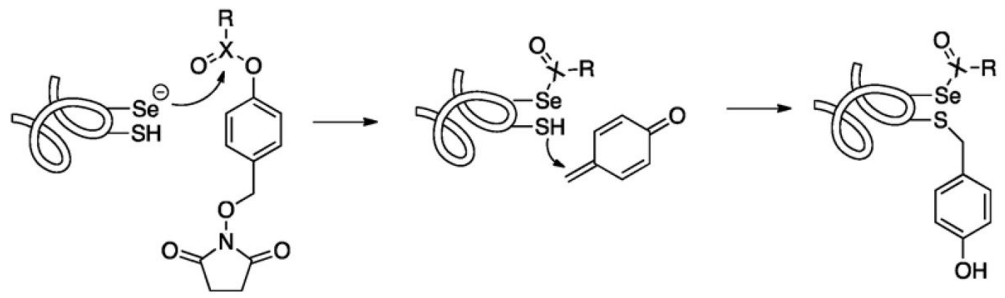

Figure 8.

Cartoon presenting a plausible mechanistic scheme for TrxR inhibition by inhibitors 6 and 3 . Note, $\mathrm{X}=\mathrm{C}$ or $\mathrm{S}$ representing $\mathbf{6}$ or $\mathbf{2 3}$, respectively. 

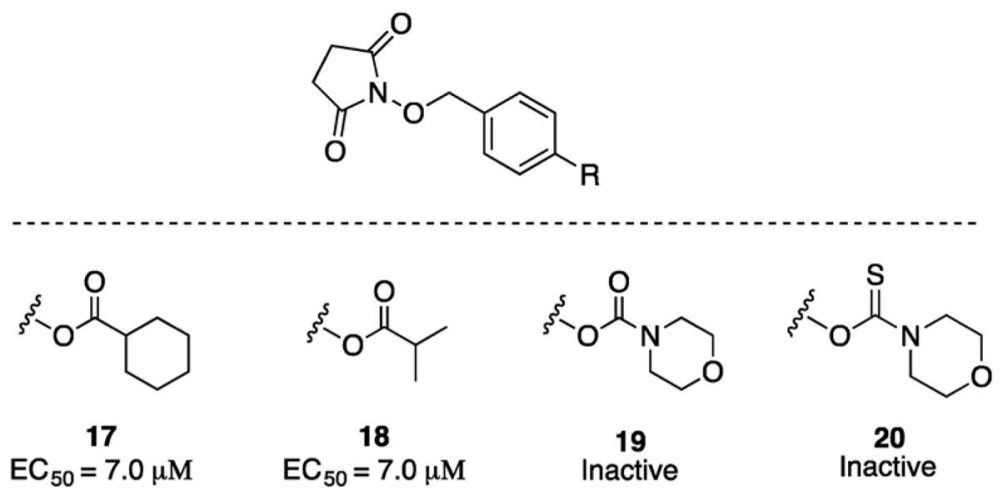

19
Inactive

20
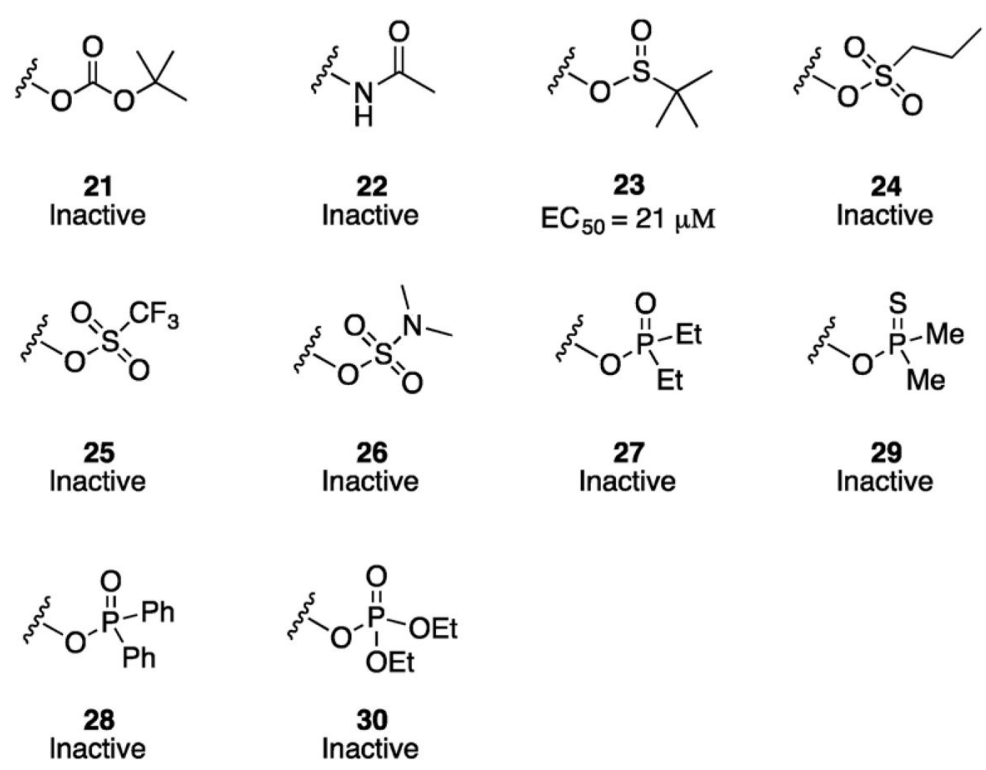

Inactive

\section{Chart 1.}

SAR Studies with Ester Congeners ${ }^{\mathrm{a}}$

${ }^{\text {aSee }}$ the Supporting Information for detailed syntheses. 


\section{Table 1}

Structures Derived from 2 and Their Cellular Activity ${ }^{a}$

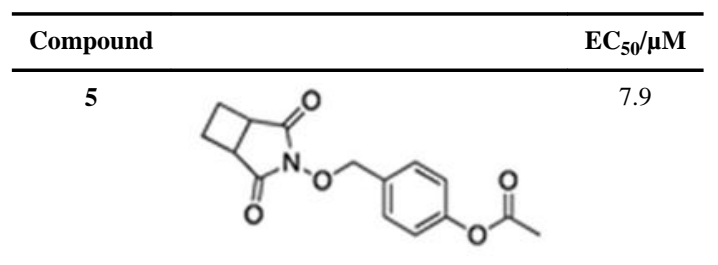

6

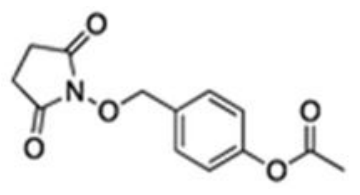

4.9

록

7

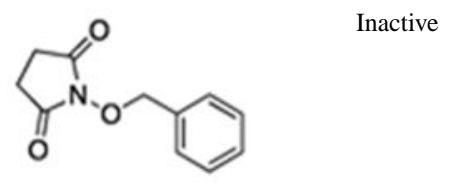

8

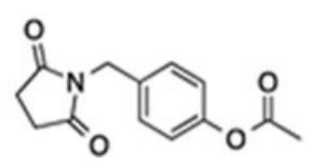

Inactive

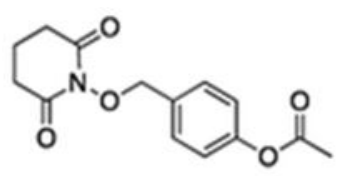

47

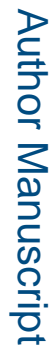

10

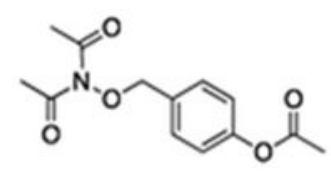

Inactive

11

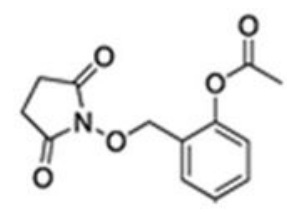

${ }^{a}$ See the Supporting Information for detailed syntheses. 
Table 2

SAR Studies for Alternative Leaving Groups Other than the NHS ${ }^{a}$

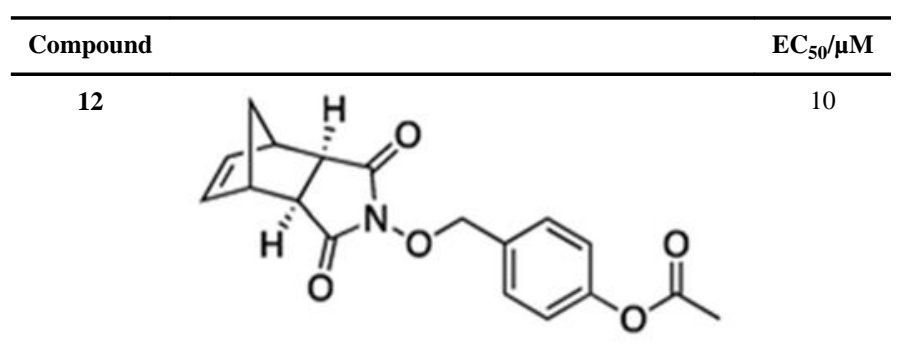

13<smiles>CC(=O)Oc1ccc(CON2C(=O)[C@@H]3C4C=CC(O4)[C@@H]3C2=O)cc1</smiles>

14<smiles>CC(=O)Oc1ccc(COn2nnc3ccccc32)cc1</smiles>

6.4

15<smiles>CC(=O)Oc1ccc(COn2nnc3cccnc32)cc1</smiles>

16

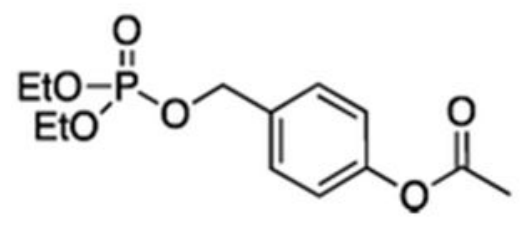

5.9

5.3

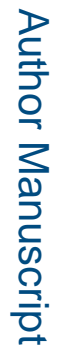

12

${ }^{a}$ See the Supporting Information for detailed syntheses. 